\title{
5-year longitudinal study of clinical and patient-reported outcomes in acute anterior uveitis
}

\author{
Christine Goodchild ${ }^{1,2} \cdot$ Micheal O'Rourke ${ }^{1,2} \cdot$ Muhammad Haroon $^{3} \cdot$ Oliver FitzGerald $\mathbb{C}^{4} \cdot$ Conor C. Murphy ${ }^{1,2}$
}

Received: 2 November 2019 / Revised: 24 April 2020 / Accepted: 27 April 2020 / Published online: 13 May 2020

(c) The Author(s), under exclusive licence to The Royal College of Ophthalmologists 2020

\begin{abstract}
Introduction Acute anterior uveitis (AAU) is a frequently encountered form of uveitis, most commonly an immunemediated condition associated with the HLA-B27 gene with or without spondyloarthritis, or idiopathic in nature. This study's aim was to measure clinical and patient-reported outcomes 5 years after the first episode of immune-mediated AAU. Methods This is a longitudinal observational study. Ninety-six patients who underwent evaluation at the time of presentation with their first episode of AAU were invited to return for evaluation 5 years later. Standardised ocular history, clinical examination and quality of life (QOL) assessment with the Short Form 36 (SF-36) and the Vision Core Measure 1 (VCM 1) questionnaire were completed and analysed.

Results Fifty-four patients (56\%) returned for subsequent assessment. Physical function was the only sub scale domain of the SF-36 that had significantly deteriorated over the 5 years since the first episode of AAU (45.95 vs. $49.37, p=0.003$ ). Only 7.4\% $(n=4)$ of patients expressed "more than a little concern" regarding their vision, reflected by a VCM1 score of 2.0 or more. At 5 years, the mean best corrected visual acuity (BCVA) of eyes affected by AAU was LogMAR 0.02 and only $3 \%(n=2)$ of eyes had a BCVA of less than Logmar of 0.3 . Five affected eyes $(8 \%)$ had developed cataract and no patients had developed glaucoma by the 5 year review.

Conclusions This study demonstrates that immune-mediated AAU has an excellent 5 year prognosis with minimal impact on patients' health and vision-related quality of life.
\end{abstract}

\section{Introduction}

Acute anterior uveitis (AAU) is the commonest form of uveitis. It accounts for $50-92 \%$ of all cases of uveitis and has an incidence of 17.4 to 52.4 cases per 100,000 personyears [1-4]. AAU can be infectious or immune-mediated in nature and the latter may be idiopathic or occur in the presence of systemic autoimmune conditions. Spondyloarthritis ( $\mathrm{SpA})$ is the most common systemic disease

Conor C. Murphy

conorcmurphy@rcsi.ie

1 Department of Ophthalmology, Royal Victoria Eye and Ear Hospital, Dublin, Ireland

2 RCSI School of Medicine, Dublin, Ireland

3 Department of Rheumatology, University Hospital Kerry, Kerry, Ireland

4 Department of Rheumatology, St. Vincent's University Hospital, Dublin, Ireland association [5]. SpA is a group of systemic disorders characterised by an inflammatory arthritis that includes psoriatic arthritis, reactive arthritis, arthritis related to inflammatory bowel disease, a subgroup of juvenile idiopathic arthritis and ankylosing spondylitis-the prototype SpA. Previous work by our group developed and validated a highly sensitive and specific algorithm, called the Dublin Uveitis Evaluation Tool (DUET), which helps to detect $\mathrm{SpA}$ in patients presenting with AAU and to streamline referrals between ophthalmologists and rheumatologists [6].

As part of the DUET study, we measured the impact of AAU and previously undiagnosed $\mathrm{SpA}$ on patient wellbeing, by using the health-related (HRQOL) and visionrelated quality of life (VRQOL) questionnaires, the Short Form 36 (SF-36) and the Vision Core Measure 1 (VCM1) [7-9]. We found that physical SF-36 scores were reduced during active AAU and improved on resolution but remained below population norms. We also reported that patients with SpA-associated AAU had a worse quality of life than those with idiopathic AAU in the acute setting [8]. 
It is clear therefore that AAU affects physical aspects of quality of life more than is apparent by measuring clinical variables alone, especially in those also affected by previously undiagnosed $\mathrm{SpA}$.

Patients with a history of AAU may experience an impaired quality of life because of concerns over sight loss, which can be caused by the development of cataract or glaucoma, pain or a reduction in physical functioning, particularly in those also affected by SpA. The long-term impact on patient well-being is poorly defined. There is a paucity of long-term prospective observational studies on the prognosis of AAU in terms of quality of life impairment and clinical outcomes, particularly in relation to sightthreatening complications of uveitis such as cataract, glaucoma and macular oedema [10-12].

The aims of the present study were: (1) to evaluate VRQOL and HRQOL in patients with idiopathic and SpAassociated AAU 5 years after they presented with their first episode of AAU, (2) to compare these to QOL outcomes at 5 years with an age-matched general population, and (3) to report on the long-term clinical outcomes following the first episode of idiopathic and SpA-associated AAU.

\section{Methods}

\section{Study design and participants}

This is a longitudinal observational study of a cohort of 96 patients who had presented with their first episode of immune-mediated AAU in 2012 and had participated at that time in the DUET study. In the DUET study, consecutive patients attending the ophthalmic emergency department with presumed non-infectious immune-mediated AAU of no known cause underwent a complete evaluation by an ophthalmologist and a rheumatologist for the purposes of developing a clinical algorithm for the early detection of spondyloarthritis [6]. Out of a total of 173 DUET study participants, 96 patients had experienced their first episode of AAU at the time of participation in 2012. DUET study participants with a prior history of AAU $(n=77)$ were excluded as the precise date of their first episode of AAU could not be ascertained. Each of these 96 patients was contacted via telephone and invited to attend a dedicated uveitis research clinic between January and August 2018 at the Royal Victoria Eye and Ear Hospital, Dublin. If initial contact was unsuccessful, patients were contacted 2 additional times via telephone on different days and a letter of invitation to attend was sent out via postal mail. The DUET study also excluded patients with a history of intraocular surgery, trauma, or a known cause of AAU such as Behcet's disease, previously diagnosed spondyloarthritis, sarcoidosis or viral uveitis.

\section{Clinical evaluation}

Ophthalmic evaluation included standard history and clinical examination for uveitis patients, including Log MAR visual acuity measurement, LOCS III assessment of cataract and OCT scanning [13]. The number of recurrences of AAU, the use of topical or systemic corticosteroids and other therapies and the diagnosis and management of related systemic diseases were recorded. Rheumatological screening for undiagnosed SpA was included as part of the assessment. Presence of new undiagnosed back pain and stiffness lasting more than $30 \mathrm{~min}$, new heel or joint pain and swelling and a diagnosis of SpA not made through the DUET study were recorded. All clinical and demographic data were collected with standardised questionnaires. Data from the DUET study were used for comparative purposes to monitor progression.

\section{Quality of life assessment}

Health-related (HRQOL) and vision-related quality of life (VRQOL) were evaluated using the United Kingdom (UK) version of the Short Form 36 (SF-36) and the Vision Core Measure 1 (VCM1) questionnaires, respectively, as these were the questionnaires used during the DUET study in 2012. The data were compared with quality of life scores recorded 5 years earlier during the DUET study at the time of active AAU and at resolution that was defined as 6 weeks after active episode. A longitudinal analysis of QOL over the course of 5 years from first onset of AAU was therefore performed.

The SF-36 consists of 36 questions grouped into eight scales: physical functioning (PF), social functioning (SC), mental health $(\mathrm{MH})$, energy/vitality $(\mathrm{EV})$, bodily pain $(\mathrm{P})$, role limitations due to physical problems (RLP), role limitations due to emotional problems (RLM), and general health perception (GH) [9, 14]. These factors are collated into the physical composite score (PCS) which is accumulated from the PF,RLP, P, and GH sub scales and the mental composite score (MCS) which is combined from the EV, SC, RLM, and MH sub scales $[14,15]$. The sub scale scores range from 0 to $100 \%$, with higher scores indicating better HRQOL [9]. To facilitate comparison with age and sex-matched general population norms, the SF-36 sub scale scores were presented as $\mathrm{T}$ scores, which were calculated using means and standard deviations from the age and sex match UK normative set. For $\mathrm{T}$ scores, normative data set has a mean of 50 and standard deviation of 10. Patient scores greater than 50 represent better than average quality of life and below 50 represent worse than average quality of life [16].

The VCM1 is a 10 item subjective questionnaire used to measure VRQOL. The questionnaire enables the patients to rate the physical, social, and psychological effects of 
performing common daily activities due to problems with their vision [8]. The scores range from 0.0 (best score) to 5.0 (worst score) and a score of $>2$ represents "more than a little" concern about vision [7, 17].

\section{Long-term clinical outcomes of AAU}

The following clinical outcomes were evaluated over the 5 year period following initial presentation with AAU: episodes of recurrence of AAU or development of chronic AU in the presenting eye, LogMar best corrected visual acuity (BCVA), cataract (defined as new posterior sub-capsular cataract assessed by LOCS III grading), macular oedema by $\mathrm{OCT}$, and development of glaucoma.

\section{Data analysis}

Descriptive statistics were reported as mean and standard deviation (SD) or number $(n)$ and percentages (\%) as appropriate to describe the demographic and clinical characteristics of the patients. All the data were collected and processed as quantitative data. Nominal data, including dichotomous data, were coded numerically to facilitate analysis.

Interpretation of SF-36 questionnaires was conducted using the standard guide [9]. Statistical analysis was performed using STATA version 15.1 software and Prism 8 for MacOS. All tests were two-tailed and statistical significance was attributed when $p<0.05$. Wilcoxon signed-rank test was used to compare SF-36 sub scales between 5 year and 6 week scores from the same patient. Independent student ttest was used for comparison between patients and normative data. For other comparisons, a Mann-Whitney $U$ test was use to test for differences in 2 unmatched samples as they were not normally distributed. A spearman correlation test was used to analyse the correlation between samples. Logistic or linear regression analysis was used for univariable or multivariable analysis depending on whether the data was categorical or continuous.

This study was approved by the Research Ethics Committee of the Royal Victoria Eye and Ear Hospital and was conducted in accordance with the Declaration of Helsinki [18]. All participants provided written informed consent. EU general data protection regulations were adhered to [19].

\section{Results}

\section{Patient characteristics}

Of the 96 eligible patients invited to take part in this study, $54(56 \%)$ patients participated. The remainder either declined participation or failed to attend for assessment. The mean time to follow up from the first episode of AAU was $5.9 \pm 0.36$ years. The majority of patients were caucasian Irish $(n=50,93 \%)$, male $(n=33,61 \%)$, had idiopathic AAU $(n=37,69 \%)$ and unilateral disease at initial presentation $(n=45,83 \%)$. Seventeen $(31 \%)$ patients were HLA-B27 positive. Thirteen (24\%) patients had SpA (all HLA-B27 positive), of whom 3 (23\%) were on immunosuppressive therapy, 3 (23\%) were on NSAIDs only and 7 (54\%) were not on any treatment for SpA. During the 5 years since initial presentation with their first episode of AAU, only $20(37 \%)$ patients experienced a recurrence of uveitis. Of the patients who had recurrences, 8 patients (40\%) had HLA-B27 positive AAU with an average of 5.3 episodes over 5 years and 12 patients $(60 \%)$ had idiopathic AAU with an average of 3.1 episodes over 5 years. Table 1 shows the individual demographic characteristics of the patients. There were only $4(8 \%)$ patients in the idiopathic AAU group who had new back pain lasting more than 30 min, of which only $2(4 \%)$ patients did not have an established diagnosis. In this group no patient had a subsequent diagnosis of SpA. The majority of patients who underwent assessment were immediately discharged from follow up $(n=44,81 \%)$. Seven (13\%) patients were referred for uveitis clinic follow up and $3(6 \%)$ patients were already regular attenders of the uveitis clinic. The three regular attenders of the clinic included two patients who had developed chronic anterior uveitis and were off all treatment and one patient who had developed late onset multifocal choroiditis requiring low dose oral prednisolone.

There were 42 (44\%) patients who were eligible for this study but did not participate. We were unable to establish contact with 27 (64\%) of patients from this group both via telephone and postal mail. Patients in this group were younger than participants ( $43 \pm 14$ vs $50 \pm 15$ years, $p=$ $0.02)$ and $13(31 \%)$ patients did not attend their 6 week appointment $(p<0.000)$. Gender and HLA-B27 status in both attendees and non-attendees groups were similar. Analysis of HRQOL and VRQOL at resolution (6 weeks) between attendees and non-attendees showed no difference aside from health perception (50.68 vs $45.29, p=0.03$ ) where attendees had a better perception of their health. Table 2 shows the demographic data of non-attendees. Table 3 compares the HRQOL and VRQOL scores at resolution between attendees and non-attendees.

\section{Quality of life}

Table 4 compares the HRQOL and VRQOL scores 5 years following initial presentation with AAU with those recorded after resolution of the inflammation at 6 weeks after initial presentation. It also compares the patients' QOL scores with those of the general age-matched population. Physical 
Table 1 Patient demographic of attendees.

\begin{tabular}{|c|c|c|}
\hline \multicolumn{2}{|l|}{ Variable } & Results \\
\hline \multicolumn{2}{|c|}{ Time to follow up (mean years \pm SD) } & $\begin{array}{l}5.9 \pm 0.36(\min = \\
5.1, \max =6.8)\end{array}$ \\
\hline \multicolumn{2}{|c|}{ Age $($ mean years $\pm \mathrm{SD})$} & $\begin{array}{l}50 \pm 15(\min =22 \\
\max =82)\end{array}$ \\
\hline & Age Male & $48 \pm 16$ \\
\hline & Age Female & $52 \pm 14$ \\
\hline \multirow[t]{2}{*}{ Sex } & Male & $33(61 \%)$ \\
\hline & Female & $21(39 \%)$ \\
\hline \multirow[t]{3}{*}{ Laterality } & Right & $23(42 \%)$ \\
\hline & Left & $22(41 \%)$ \\
\hline & Bilateral & $9(17 \%)$ \\
\hline HLA-B27 & Positive & $17(31 \%)$ \\
\hline \multirow[t]{4}{*}{ SpA } & Positive & $13(24 \%)$ \\
\hline & On immunosuppressant & $3(23 \%)$ \\
\hline & On NSAIDs only & $3(23 \%)$ \\
\hline & No treatment & $7(54 \%)$ \\
\hline \multicolumn{2}{|l|}{ Idiopathic AAU } & $37(69 \%)$ \\
\hline \multirow[t]{4}{*}{ Ethnicity } & Caucasian-Irish & $50(93 \%)$ \\
\hline & Caucasian-Non-Irish & $1(2 \%)$ \\
\hline & Black & $1(2 \%)$ \\
\hline & Asian & $2(4 \%)$ \\
\hline \multirow[t]{3}{*}{ Recurrence of AAU } & Total & $20(37 \%)$ \\
\hline & HLA B27 Positive & $\begin{array}{l}8(40 \%) \text { Ave } 1.07 \\
\text { episodes per year }\end{array}$ \\
\hline & Idiopathic & $\begin{array}{l}12(60 \%) \text { Ave } 0.62 \\
\text { episodes per year }\end{array}$ \\
\hline
\end{tabular}

The clinical and demographic details of patients at 5 years post presentation for acute anterior uveitis $(n=54)$.

function was the only sub scale domain of the SF-36 that had significantly deteriorated over the 5 years since the first episode of AAU (45.95 vs. 49.37, $p=0.003$ ). Physical function was also the only SF-36 sub scale that was lower than the general age-matched population ( 45.95 vs. 50.00, $p=0.03)$. There was no significant change in the VCM1 score between 6 weeks and 5 years, indicating that patients had minimal concern about their vision, both 6 weeks following first episode of AAU $(\mathrm{VCM} 1=0.74)$ and 5 years later $(\mathrm{VCM} 1=0.52, p=0.10)$. A VCM1 score of 2 and above ("more than a little concern about vision") was seen in only $4(7.4 \%)$ patients as demonstrated in Fig. 1. There was a moderate correlation between the Physical Component Score (PCS) and the mental component score (MCS) and the VCM1 score (PCS, $r=-0.46, p<$ 0.001 ; MCS, $r=-0.41, p=0.002)$. Therefore, better VCM1 scores were associated with better PCS and MCS scores.

An analysis of HRQOL and VRQOL 5 years following the first episode of AAU for patients with SpA associated
Table 2 Patient demographic of non-attendees.

\begin{tabular}{lll}
\hline Variable & & Results \\
\hline Age (mean years \pm SD) & $43 \pm 14(\min =$ \\
& & $20, \max =87)$ \\
& Age Male & $43 \pm 11$ \\
& Age Female & $43 \pm 18$ \\
Sex & Male & $24(57 \%)$ \\
Reason for not & Female & $18(43 \%)$ \\
attending & Unable to establish contact via & $27(64 \%)$ \\
& telephone or post & \\
& Failure to attend OPD after & $6(14 \%)$ \\
& contact and appointment & \\
HLA-B27 & was made & $9(22 \%)$ \\
Ethnicity & Refused to participate & $14(33 \%)$ \\
& Positive & $33(79 \%)$ \\
& Caucasian-Irish & $2(5 \%)$ \\
& Caucasian-Non-Irish & $1(2 \%)$ \\
& Black & $6(14 \%)$
\end{tabular}

The clinical and demographic details of patients who were eligible for the study but did not participate in the 5 year follow up $(n=42)$.

Table 3 SF-36 and VCM1 scores comparing attendees to nonattendees scores at time of resolution.

\begin{tabular}{|c|c|c|c|c|c|}
\hline \multirow[t]{2}{*}{ Variable } & \multicolumn{2}{|c|}{ Attendees } & \multicolumn{2}{|c|}{$\begin{array}{l}\text { Non- } \\
\text { attendees }\end{array}$} & \multirow[b]{2}{*}{$P$ value } \\
\hline & Mean & SD & Mean & SD & \\
\hline Physical Function & 49.37 & 12.77 & 50.87 & 10.12 & 0.44 \\
\hline Social Function & 47.43 & 12.29 & 48.30 & 10.64 & 0.83 \\
\hline Mental Health & 49.16 & 9.82 & 45.23 & 13.66 & 0.28 \\
\hline Pain & 47.90 & 10.10 & 47.21 & 10.11 & 0.87 \\
\hline Role limitation—Physical & 46.93 & 11.95 & 45.29 & 16.60 & 0.77 \\
\hline Role limitation-Mental & 48.31 & 13.32 & 48.22 & 14.88 & 0.66 \\
\hline Energy/Vitality & 48.27 & 14.29 & 48.16 & 12.18 & 0.80 \\
\hline Health perceptions & 50.68 & 12.32 & 45.29 & 11.48 & $\mathbf{0 . 0 3}$ \\
\hline Physical composite score & 48.71 & 9.08 & 47.17 & 8.68 & 0.31 \\
\hline Mental composite score & 48.29 & 9.57 & 47.48 & 10.52 & 0.60 \\
\hline VCM 1 & 0.74 & 0.98 & 0.78 & 0.79 & 0.34 \\
\hline
\end{tabular}

SF-36 sub scale $\mathrm{T}$ scores for patients who participated in this study $(n=54)$ compared with those who did not $(n=42)$. All participants completed QOL questionnaires at 6 weeks compared with only 29 $(69 \%)$ of non-attendees $(p<0.001)$. Health perception was statistically significantly lower in non-attendees $(p=0.03)$.

Significant data are highlighted in bold. SF-36: Medical Outcomes Study Short Form-36.

uveitis and non-SpA associated uveitis was performed. There were no significant differences in any of the SF-36 sub scales, including PCS $(p=0.65)$ and MCS $(p=0.51)$, or VCM1 $(p=0.66)$ scores at 5 years between AAU patients with and without SpA (Table 5). However, when compared with the age-matched general population, patients 
Table 4 SF-36 and

VCM1 scores for all AAU

patients comparing 5 year scores to general population and scores at time of resolution.

\begin{tabular}{|c|c|c|c|c|c|c|c|c|c|c|}
\hline \multirow[t]{2}{*}{ Variable } & \multicolumn{2}{|c|}{$\begin{array}{l}\text { General } \\
\text { population }\end{array}$} & \multicolumn{2}{|c|}{5 years } & \multirow[b]{2}{*}{$P$ value } & \multicolumn{2}{|c|}{ Resolution } & \multicolumn{2}{|l|}{ 5years } & \multirow[b]{2}{*}{$P$ value } \\
\hline & Mean & SD & Mean & SD & & Mean & $\mathrm{SD}$ & Mean & SD & \\
\hline Physical function & 50.00 & 10.00 & 45.95 & 9.44 & $0.03 *$ & 49.37 & 12.77 & 45.95 & 9.44 & $0.003 * *$ \\
\hline Social Function & 50.00 & 10.00 & 48.10 & 12.26 & 0.38 & 47.43 & 12.29 & 48.10 & 12.26 & 0.91 \\
\hline Mental Health & 50.00 & 10.00 & 50.64 & 11.10 & 0.75 & 49.16 & 9.82 & 50.64 & 11.10 & 0.31 \\
\hline Pain & 50.00 & 10.00 & 48.94 & 9.56 & 0.57 & 47.90 & 10.10 & 48.94 & 9.56 & 0.52 \\
\hline Role limitation-Physical & 50.00 & 10.00 & 49.56 & 13.11 & 0.85 & 46.93 & 11.95 & 49.56 & 13.11 & 0.05 \\
\hline Role limitation-Mental & 50.00 & 10.00 & 49.36 & 15.62 & 0.80 & 48.31 & 13.32 & 49.36 & 15.62 & 0.80 \\
\hline Energy/Vitality & 50.00 & 10.00 & 48.97 & 12.31 & 0.63 & 48.27 & 14.29 & 48.97 & 12.31 & 0.77 \\
\hline Health perceptions & 50.00 & 10.00 & 49.17 & 12.04 & 0.69 & 50.68 & 12.32 & 49.17 & 12.04 & 0.45 \\
\hline Physical composite score & 50.00 & 10.00 & 48.40 & 9.15 & 0.39 & 48.71 & 9.08 & 48.40 & 9.15 & 0.48 \\
\hline Mental composite score & 50.00 & 10.00 & 49.27 & 11.57 & 0.72 & 48.29 & 9.57 & 49.27 & 11.57 & 0.51 \\
\hline VCM 1 & & & & & & 0.74 & 0.98 & 0.52 & 0.75 & 0.10 \\
\hline
\end{tabular}

SF-36 sub scale T scores for all patients with AAU $(n=54)$ at 5 years post initial episode and resolution of AAU (6 weeks) compared with an age- and sex-matched population using UK normative data.

A significant decline was seen for physical function $(p=0.003)$ when T-scores were compared over time. Significant data are highlighted in bold. SF-36: Medical Outcomes Study Short Form-36.

*Compared with the general population. **Compared with 6 weeks post initial episode of AAU after it had resolved.

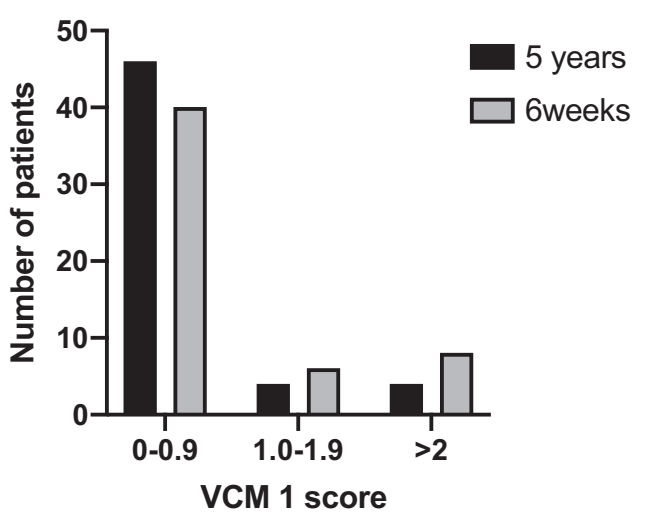

Fig. 1 Comparison of VCM 1 scores 6 weeks and 5 years after presentation with the first episode of AAU. Patients have a better VCM 1 score at 5 years compared with scores at 6 weeks post presentation. At 5 years only $7.4 \%(n=4)$ of patients versus $14.8 \%(n=$ 8) at 6 weeks expressed "more than a little concern" over their vision which is reflected by a VCM1 score of 2.0 or more.

with AAU not associated with SpA had a reduced physical function score (45.53 vs. $50.00, p=0.046)$ but patients with AAU associated with SpA did not.

\section{Long-term clinical outcomes of AAU}

LogMAR BCVA was $0.02 \pm 0.14$ for all eyes with a history of AAU ( $n=63$ as 9 patients had bilateral AAU) and $0.07 \pm 0.36$ $(p=0.82)$ in eyes not affected by AAU $(n=45)$. There were no patients with a recorded BCVA of less than LogMAR 1 (Snellen equivalent 20/200). Two eyes (3\%) affected by AAU had a BCVA of less than Logmar of 0.3 (Snellen equivalent 20/
Table 5 SF-36 and VCM 1 scores for patients with and without SpA.

\begin{tabular}{|c|c|c|c|c|c|}
\hline \multirow[t]{2}{*}{ Variables } & \multicolumn{2}{|l|}{ SpA } & \multicolumn{2}{|c|}{ Non-SpA } & \multirow[b]{2}{*}{$P$ value } \\
\hline & Mean & SD & Mean & SD & \\
\hline Physical function & 47.30 & 7.58 & 45.53 & 10.00 & 0.67 \\
\hline Social function & 46.53 & 14.62 & 48.60 & 11.58 & 0.88 \\
\hline Mental health & 47.00 & 17.92 & 51.79 & 7.83 & 0.70 \\
\hline Pain & 49.44 & 8.69 & 48.78 & 9.91 & 0.96 \\
\hline Role limitation-physical & 48.83 & 12.11 & 49.79 & 13.55 & 0.45 \\
\hline Role limitation-mental & 42.69 & 26.92 & 51.47 & 9.30 & 0.18 \\
\hline Energy/Vitality & 47.65 & 14.26 & 49.39 & 11.79 & 0.75 \\
\hline Health perceptions & 52.44 & 8.96 & 48.13 & 12.78 & 0.29 \\
\hline Physical composite score & 49.50 & 7.73 & 48.05 & 9.61 & 0.65 \\
\hline Mental composite score & 45.97 & 17.86 & 50.31 & 8.78 & 0.51 \\
\hline VCM 1 & 0.48 & 0.54 & 0.54 & 0.81 & 0.66 \\
\hline
\end{tabular}

SF-36 sub scale T scores for the subgroup of patients SpA associated uveitis $(n=13)$ and non-SpA associated uveitis $(n=41)$. There was no statistically significant difference found.

SF-36: Medical Outcomes Study Short Form-36. SpA: Spondyloarthritis.

40). Thirty-six eyes (57\%) affected by AAU had a final BCVA of equal to or better than LogMAR 0 (Snellen equivalent 20/ 20). A weak correlation was found between BCVA and SF-36 sub scale scores for physical function $(r=-0.41, p=0.002)$, mental health $(r=-0.36, p=0.008)$, role limitation-physical $(r=-0.43, p=0.001)$, general health perception $(r=-0.34$, $p=0.01)$ and energy and vitality $(r=-0.30, p=0.03)$. Therefore, better BCVA was associated with better HRQOL sub-scale scores which means patients who have better visual acuity are more likely to have a better quality of life. 
Posterior sub-capsular cataract developed in $5(8 \%)$ eyes affected by AAU and $1(2 \%)$ eye unaffected by uveitis ( $p=$ $0.39)$. BCVA in eyes with a history of AAU with cataract was LogMAR $0.03 \pm 0.12$ and BCVA in eyes without cataract was $0.01 \pm 0.14(p=0.56)$, indicating minimal effect on vision. There was no correlation between cataract formation and QOL. Cataract formation was not significantly associated with age, HLA B27 status or the intensity or duration of topical corticosteroid treatment during the initial episode of AAU by regression analysis. None of the patients had cystoid macular oedema, elevated intraocular pressures or a diagnosis of glaucoma.

\section{Discussion}

This study of self-reported and clinical outcomes 5 years after the first episode of idiopathic or spondyloarthritisrelated AAU confirms an excellent long-term prognosis for this form of uveitis. Patients maintained excellent visual function in terms of both visual acuity and VRQOL using the established VCM1 questionnaire, through which they reported minimal concern over their vision. Only $7.4 \%$ $(n=4)$ of patients expressed "more than a little concern" regarding their vision, which is reflected by a score of 2.0 or more. This is a lower figure than that the perviously reported rate of $28 \%$ of patients with chronic uveitis in a study by Jalil et al. [20]. The only HRQOL domain affected in the long-term was physical functioning, which was worse 5 years after the first episode of AAU, than it was 6 weeks after presentation following resolution of the inflammation. This same domain was also worse at 5 years, than the agematched general population. However, it is reassuring to report that QOL is as well maintained in those affected by $\mathrm{SpA}$-associated AAU as those with idiopathic AAU 5 years following diagnosis. This supports the evidence that early identification and treatment of $\mathrm{SpA}$ can contribute to maintaining a good quality of life [21]. In addition, none of the patients in the idiopathic AAU group were subsequently diagnosed with SpA which adds to the validity and usefulness of the DUET algorithm in the early detection of SpA in patients presenting with AAU.

This cohort of patients is a subgroup of participants of the DUET study, in which we comprehensively evaluated QOL during AAU and 6 weeks later following resolution of the inflammation. During active inflammation, all physical domains of the SF-36 were significantly impaired, as was social functioning and the physical and MCSs. These improved significantly by the time of complete resolution of AAU and, as demonstrated in the present follow up study at 5 years or more, returned to normal except for physical functioning. It is notable that the decrease in physical functioning scores compared with the general population was found for the idiopathic AAU cohort but not the SpAassociated AAU cohort. This surprising finding might reflect the low numbers in each category in this study, or alternatively the normal physical functioning of the SpA cohort might reflect well controlled systemic disease in these patients. A reduction in physical function in patients with AAU was also demonstrated in an Italian population by Fabiani et al. They reported that patients with AAU have lower physical function scores compared with healthy controls and also compared with patients with panuveitis [22]. A general reduction in HRQOL and VRQOL in patients with uveitis compared with the general population is reported in a study by Schiffman et al. However in this paper the majority of patients $(83 \%)$ had uveitis involving the posterior segment [23]. Murphy et al. similarly reported a significant reduction in SF-36 scores for the sub scales social functioning, general health perception and pain in patients with intermediate uveitis [24]. A study by Silva et al. showed that in a Brazilian population, patients with uveitis had lower HRQOL scores in all sub scales and that poor physical functioning scores were associated with lower family income [25].

The clinical outcomes reported in this study indicate that idiopathic and SpA-associated AAU have an excellent prognosis in the long-term. The mean BCVA was LogMAR 0.02 and $57 \%(n=36)$ of patients had a BCVA of 0.00 or better in eyes affected by uveitis. None of the patients had severe visual impairment (LogMAR $>1$, Snellen BCVA > $20 / 200$ ) in either eye, in contrast to other studies of anterior uveitis in which this occurs in $2-11 \%$ of patients [10-12]. Posterior sub capsular cataracts occurred in $8 \%(n=5)$ of eyes affected by AAU compared with $2 \%(n=1)$ of unaffected eyes. However, the development of cataract did not significantly affect BCVA when compared with eyes unaffected by cataract, indicating that it was mild and nonsignificant in all patients. The rate of cataract development in AAU eyes in this study was similar to the rate reported by the National Eye Institute in the US in the general population. They found the prevalence of cataracts to be 5 to $25 \%$, in patients aged 50 to 69 years old, with no history of uveitis [26]. Cataract formation, in the present study, was not associated with increasing age or HLA-B27 status. In contrast, a study by Power et al. showed that patients who have HLA-B27 positive AAU have twice the likelihood of developing cataract [12].

None of the patients in this study had glaucoma 5 years after their first presentation with AAU. In larger studies, the rate of secondary glaucoma development is 5 to $14 \%$ depending on HLA-B27 and SpA status [11, 12].

The key strengths of this study are that it is a 5 year longitudinal evaluation of patient reported and clinical outcomes in the commonest form of uveitis and that it builds on two previous reports on this cohort of patients from the 
DUET study [3, 9]. In addition, in contrast to other uveitis studies we recruited patients without an underlying systemic diagnosis through the emergency department as opposed to specialist uveitis clinics $[12,20,23,24,27]$. This method of recruitment allowed us to observe and evaluate the evolution of quality of life over a 5 year period, improving our understanding of the long term impact of AAU on patients from the time of presentation. However, as patients with associated systemic diseases were excluded, our study might also have excluded patients with more severe uveitis. Another limitation of this study was the loss to follow up of $44 \%$ of the patients from the initial DUET cohort. The study might have been underpowered to detect small differences because of the small sample size, resulting in a type II error. Suboptimal response rates with this type of research have been documented previously, especially in the working age population and the younger age of non-attenders compared with participants in this study was notable [28]. Finally, this study had a homogenous patient population of a majority of caucasian patients (95\%) which could limit its external validity in populations of different ethnicities.

In conclusion, this study demonstrates that both idiopathic and SpA-related AAU have an excellent 5 year prognosis with minimal impact on patients' health and VRQOL.

\section{Summary}

\section{What was known before}

- The majority of previous studies measuring the prognosis of AAU in patients with HLA-B27 AAU and idiopathic AAU focus on ocular outcomes rather than quality of life and patient reported outcomes.

- These studies are predominantly retrospective in nature with a limited follow up period.

\section{What this study adds}

- Long-term vision-related and health-related quality of life outcomes in patients with idiopathic and spondyloarthritisrelated acute anterior uveitis are reported.

- Long-term clinical outcomes, including visual acuity, cataract and glaucoma formation, are reported for patients with non-infectious acute anterior uveitis.

- This study also expands on the prior research conducted and published by the same research group (O'Rourke M, Haroon $\mathrm{M}$ et al.) as part of the DUET (Dublin Uveitis Evaluation Tool) study, which was published in the Journal of Rheumatology in December 2017 and the Annals of the Rheumatic Diseases in 2015.

\section{Compliance with ethical standards}

Conflict of interest The authors declare that they have no conflict of interest.

Publisher's note Springer Nature remains neutral with regard to jurisdictional claims in published maps and institutional affiliations.

\section{References}

1. Gritz DC, Wong IG. Incidence and prevalence of uveitis in Northern California; the Northern California Epidemiology of Uveitis Study. Ophthalmology. 2004;111:491-500. discussion

2. Darrell RW, Wagener HP, Kurland LT. Epidemiology of uveitis. Incidence and prevalence in a small urban community. Arch Ophthalmol (Chic, Ill: 1960). 1962;68:502-14.

3. Acharya NR, Tham VM, Esterberg E, Borkar DS, Parker JV, Vinoya AC, et al. Incidence and prevalence of uveitis: results from the pacific ocular inflammation study. JAMA Ophthalmol. 2013;131:1405-12.

4. Suhler EB, Lloyd MJ, Choi D, Rosenbaum JT, Austin DF. Incidence and prevalence of uveitis in veterans affairs medical centers of the Pacific Northwest. Am J Ophthalmol. 2008;146:890-6.

5. Chang JH, McCluskey PJ, Wakefield D. Acute anterior uveitis and HLA-B27. Surv Ophthalmol. 2005;50:364-88.

6. Haroon M, O’Rourke M, Ramasamy P, Murphy CC, FitzGerald O. A novel evidence-based detection of undiagnosed spondyloarthritis in patients presenting with acute anterior uveitis: the DUET (Dublin Uveitis Evaluation Tool). Ann Rheum Dis. 2015;74:1990-5.

7. Frost NA, Sparrow JM, Durant JS, Donovan JL, Peters TJ, Brookes ST. Development of a questionnaire for measurement of vision-related quality of life. Ophthalmic Epidemiol. 1998;5: 185-210.

8. O'Rourke M, Haroon M, Alfarasy S, Ramasamy P, FitzGerald O, Murphy CC. The Effect of Anterior Uveitis and Previously Undiagnosed Spondyloarthritis: Results from the DUET Cohort. J Rheumatol. 2017;44:1347-54.

9. Ware J, Snoww K, Ma K, Bg G SF36 Health Survey: manual and Interpretation Guide. 301993. p. 6:1-6:22, 8:1-8:5, 10:1-10:2.

10. Park SC, Ham DI. Clinical features and prognosis of HLA-B27 positive and negative anterior uveitis in a Korean population. J Korean Med Sci. 2009;24:722-8.

11. Linssen A, Meenken C. Outcomes of HLA-B27-positive and HLA-B27-negative acute anterior uveitis. Am J Ophthalmol. 1995;120:351-61.

12. Power WJ, Rodriguez A, Pedroza-Seres M, Foster CS. Outcomes in anterior uveitis associated with the HLA-B27 haplotype. Ophthalmology. 1998;105:1646-51.

13. Chylack LT Jr, Wolfe JK, Singer DM, Leske MC, Bullimore MA, Bailey IL, et al. The lens opacities classification system III. The longitudinal study of cataract study group. Arch Ophthalmol (Chic, Ill: 1960) 1993;111:831-6.

14. Ware JE Jr. SF-36 health survey update. Spine 2000;25:3130-9.

15. Jenkinson C. The SF-36 physical and mental health summary measures: an example of how to interpret scores. J health Serv Res Policy. 1998;3:92-6.

16. Jenkinson C, Coulter A, Wright L. Short form 36 (SF36) health survey questionnaire: normative data for adults of working age. BMJ: Br Med J. 1993;306:1437-40.

17. Frost A, Eachus J, Sparrow J, Peters TJ, Hopper C, Davey-Smith $\mathrm{G}$, et al. Vision-related quality of life impairment in an elderly UK population: associations with age, sex, social class and material deprivation. Eye (Lond, Engl). 2001;15(Pt 6):739-44. 
18. World Medical Association Declaration of Helsinki: ethical principles for medical research involving human subjects. JAMA. 2013;310:2191-4.

19. R Regulation (EU) $2016 / 679$ of the European Parliament and of the Council of 27 April 2016 on the protection of natural persons with regard to the processing of personal data and on the free movement of such data, and repealing Directive 95/46/EC (General Data Protection Regulation)\}. Off J Eur Union. 2016;L119:1-88.

20. Jalil A, Yin K, Coyle L, Harper R, Jones NP. Vision-related quality of life and employment status in patients with uveitis of working age: a prospective study. Ocul Immunol Inflamm. 2012;20:262-5.

21. Gran JT, Skomsvoll JF. The outcome of ankylosing spondylitis: a study of 100 patients. Br J Rheumatol. 1997;36:766-71.

22. Fabiani C, Vitale A, Orlando I, Capozzoli M, Fusco F, Rana F, et al. Impact of uveitis on quality of life: a prospective study from a tertiary referral rheumatology-ophthalmology collaborative uveitis center in Italy. Isr Med Assoc J. 2017;19:478-83.

23. Schiffman RM, Jacobsen G, Whitcup SM. Visual functioning and general health status in patients with uveitis. Arch Ophthalmol. 2001;119:841-9.
24. Murphy CC, Hughes EH, Frost NA, Dick AD. Quality of life and visual function in patients with intermediate uveitis. Br J Ophthalmol. 2005;89:1161-5.

25. Silva LMP, Arantes TE, Casaroli-Marano R, Vaz T, Belfort R, Jr., Muccioli C. Quality of Life and psychological aspects in patients with visual impairment secondary to uveitis: a clinical study in a tertiary care hospital in Brazil. Ocul Immunol Inflamm. 2019;27: 99-107.

26. Institute NE 2010 U.S. Age-specific prevalence rates for cataract by age, and race/ethnicity [webpage]. 2010. https://www.nei.nih. gov/learn-about-eye-health/resources-for-health-educators/eyehealth-data-and-statistics/cataract-data-and-statistics.

27. Miserocchi E, Modorati G, Mosconi P, Colucci A, Bandello F. Quality of life in patients with uveitis on chronic systemic immunosuppressive treatment. Ocul Immunol Inflamm. 2010;18: 297-304.

28. Pavlik VN, Hyman DJ, Vallbona C, Dunn JK, Louis K, Dewey $\mathrm{CM}$, et al. Response rates to random digit dialing for recruiting participants to an onsite health study. Public health Rep. (Wash, DC: 1974). 1996;111:444-50. 